
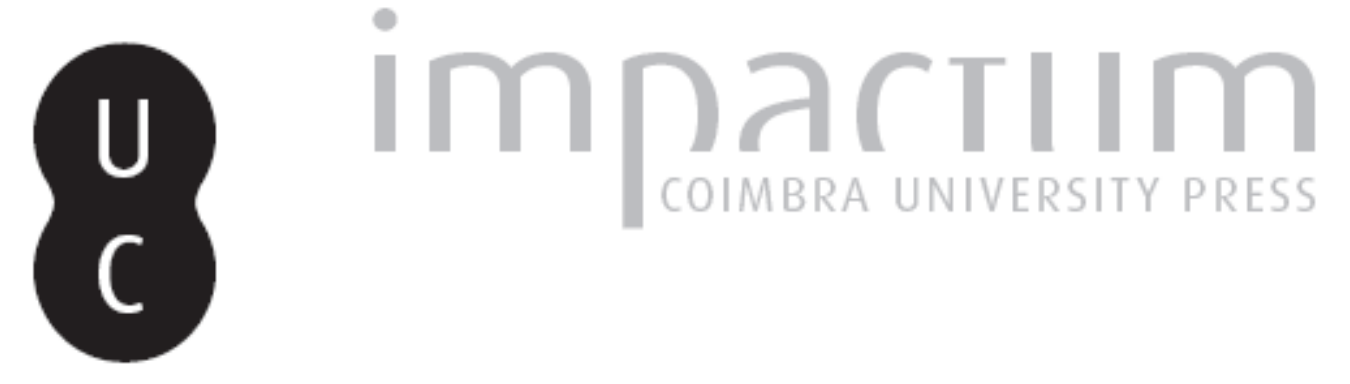

\title{
[Recensão a] Tabucchi o del Novecento, a cura di Vincenzo Russo
}

\author{
Autor(es): Capecchi, Giovanni
}

Publicado por: Imprensa da Universidade de Coimbra

URL persistente:

URl:http://hdl.handle.net/10316.2/42417

DOI:

DOI:https://doi.org/10.14195/0870-8584_9_11

Accessed : $\quad$ 26-Apr-2023 13:29:43

A navegação consulta e descarregamento dos títulos inseridos nas Bibliotecas Digitais UC Digitalis, UC Pombalina e UC Impactum, pressupõem a aceitação plena e sem reservas dos Termos e Condições de Uso destas Bibliotecas Digitais, disponíveis em https://digitalis.uc.pt/pt-pt/termos.

Conforme exposto nos referidos Termos e Condições de Uso, o descarregamento de títulos de acesso restrito requer uma licença válida de autorização devendo o utilizador aceder ao(s) documento(s) a partir de um endereço de IP da instituição detentora da supramencionada licença.

Ao utilizador é apenas permitido o descarregamento para uso pessoal, pelo que o emprego do(s) título(s) descarregado(s) para outro fim, designadamente comercial, carece de autorização do respetivo autor ou editor da obra.

Na medida em que todas as obras da UC Digitalis se encontram protegidas pelo Código do Direito de Autor e Direitos Conexos e demais legislação aplicável, toda a cópia, parcial ou total, deste documento, nos casos em que é legalmente admitida, deverá conter ou fazer-se acompanhar por este aviso.

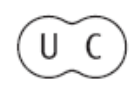




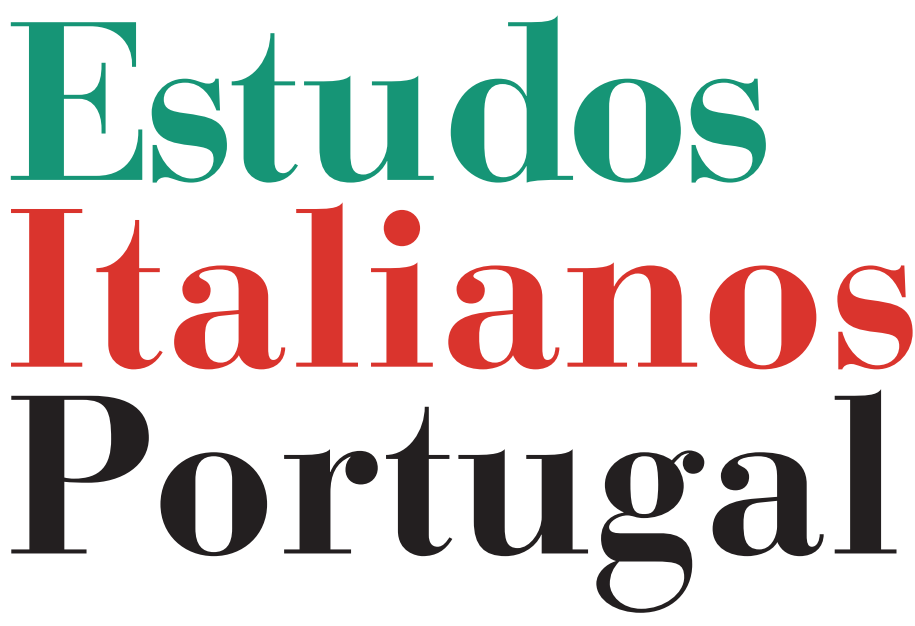

Instituto

Italiano

de Cultura

de Lisboa

Nova Série

$\mathbf{N}^{\circ} 9$ 
ve como exemplo a afirmação de que Barranco de cegos é um romance onde se pode surpreender "un tradimento dei canoni neorealisti in Alves Redol" (p. 33), sem considerar a evolução e o carácter dinâmico do movimento, o qual não obedeceu, de modo nenhum, a um esquema mecânico e com formas e fórmulas fixas. Os estudos de António Pedro Pita, de Vítor Viçoso ou de Paula Morão para o neo-realismo português, bem como os de Maria Corti sobre o neo-realismo italiano, vieram confirmar este ponto de vista. MANUEL G. SIMÕES

Tabucchi o del Novecento, a cura di Vincenzo Russo, Dipartimento di Lingue e Letterature Straniere - Facoltà di Studi Umanistici Università degli Studi di Milano, collana "di/segni" (volume n. 6), 2013, pp. 129.

Il 2013 è stato un anno importante per quanto riguarda Antonio Tabucchi, lo scrittore più europeo e meno nazionale che la letteratura italiana abbia avuto nell'ultimo trentennio, la voce "civile" più coraggiosa dopo la scomparsa di Pierpaolo Pasolini e di Leonardo Sciascia, il narratore che si è concentrato sulla Storia (e, in particolare, sui periodi in cui la libertà è stata negata e sopraffatta) e sulle storie (con i suoi personaggi-ricercatori, che si muovono in una realtà inconoscibile, che avanzano, brancolando, verso il filo dell'orizzonte che si allontana progressivamente). Nel corso del 2013, infatti, primo anno solare in assenza dello scrittore, è stato pubblicato da Feltrinelli Per Isa$b e l$, un romanzo lentamente sedimentato, dettato nel 1996, messo da parte e poi ripreso nel $2011 \mathrm{ma}$ destinato ad essere postumo, e per i tipi della stessa casa editrice e a cura di Anna Dolfi - è uscito Di tutto resta un poco, raccolta di scritti tabucchiani sulla letteratura e sul cinema, un volume denso di riflessioni sul ruolo politico della scrittura, con pagine dedicate ai libri propri e altrui, alle letture e alle riletture di una vita. Nel corso dello stesso 2013, Andrea Bajani ha pubblicato il bellissimo $M i$ riconosci, congedo da Tabucchi e omaggio ad un amico di una generazione diversa, storia degli incontri e del definitivo addio tra due scrittori che, giorno dopo giorno, hanno scoperto le proprie affinità, e, tra i volumi che studiosi e amici hanno voluto dedicare all'autore di Notturno indiano, è stato pub- 
blicato, a cura di Vincenzo Russo, Tabucchi o del Novecento, raccolta di contributi che sono nati da un'occasione ben precisa (per lo più si tratta di relazioni presentate da docenti dell'Università degli Studi di Milano nel corso di un convegno svoltosi il 21 febbraio 2013) ma che, anche rispetto ad altri "omaggi " apparsi dopo la scomparsa di Tabucchi, occupa un posto rilevante e autonomo: si presenta, innanzi tutto, come un serio approfondimento critico (lasciando sullo sfondo il ricordo dell'amico Tabucchi, che domina invece altre pubblicazioni, proprio per questo più "di occasione") e concentra l'attenzione sul rapporto tra Tabucchi e il Portogallo o, meglio ancora, su Tabucchi, la penisola iberica e la lingua portoghese.

Vincenzo Russo apre la serie e l'intero volume - analizzando il saggismo pessoano di Tabucchi, a partire dall'antologia einaudiana apparsa nel 1971 e dedicata ai poeti surrealisti portoghesi (antologia nella quale Pessoa veniva indicato come precursore dell'estetica surrealista) e svincolando questo aspetto dell'opera di Tabucchi dalla "sudditanza" nei confronti del narratore: "il saggismo tabucchia- no non è semplicemente origine né tantomeno conseguenza dell'opera narrativa"; Alessandra Cioccarelli si occupa della trilogia portoghese, analizzata partendo dal paratesto, sicuramente caratterizzata dal passaggio da una narrazione a dominante onirica e fantastica (Requiem, che ha per sottotitolo, non a caso, "un'allucinazione") ai romanzi più spiccatamente storicorealistici (Sostiene Pereira e La testa perduta di Damasceno Monteiro), che tuttavia non sembrano rappresentare l'approdo di una storia narrativa che, fuori dal Portogallo e dopo il 1997, avrebbe portato, per esempio, alla scrittura di Tristano muore ("un delirio", per ricordare il sottotitolo, sul confine tra storia e allucinazione); Davide Bigalli analizza da una prospettiva filosofica gli studi di Tabucchi su Pessoa, tra spunti esoterici (evidenziati attraverso la centralità di $O$ Marinheiro) e fallimento dei progetti illuministico e positivistico, nonché della centralità rivendicata dalla scienza; Danilo Manera svolge un percorso su Tabucchi e la Spagna, ricordando i personaggi spagnoli presenti nelle pagine tabucchiane ( $\mathrm{da}$ Francisco Goya e Federico García Lorca in Sogni di sogni a Dolores Ibarruri in Il gioco del rovescio), gli 
studi su Tabucchi e le traduzioni dei suoi libri in Spagna (con Carlos Gumpert che, oltre a tradurlo, ha anche registrato una importante intervista con l'autore) e i rapporti con scrittori spagnoli contemporanei (tra i quali spicca Enrique Vila-Matas, dichiaratamente debitore nei confronti di Donna di Porto Pim, con il quale Tabucchi ha intrecciato una amicizia fatta di "autodefinizioni condivise" e di omaggi reciproci); Roberto Vecchi si occupa di Tabucchi e del Brasile, prestando una particolare attenzione agli autori brasiliani tradotti da Tabucchi, tra fedeltà all'originale e attenzione all'eleganza della prosa italiana di arrivo, con un itinerario che conduce dalla versione datata 1974 di Il treno di Recife di José Lins do Rego alla personalissima antologia rappresentata dai trentasette testi di Carlos Drummond de Andrade, scelti e resi in italiano in Sentimento del mondo; Roberto Mulinacci si occupa infine di un episodio dimenticato ma assai importante di autotraduzione, vale a dire del capitolo quinto di Requiem, romanzo scritto in portoghese e tradotto - limitatamente alle pagine riguardanti la visita alle Tentazioni di Sant'Antonio, dipinto di Hieronymus Bosch conservato nel Museu de Arte Antiga di Lisbona, un particolare del quale è significativamente riprodotto nella copertina di questa raccolta di saggi - dallo stesso Tabucchi per i lettori del Corriere della Sera: una traduzione apparsa l'11 agosto 1991 sul quotidiano milanese, firmata con lo pseudonimo di Nuno Pereira e immediatamente rifiutata dall'autore del romanzo, che decideva poi di affidare la versione italiana dell'intera storia a Sergio Vecchio.

Ma nel volume curato da Vincenzo Russo, che si concentra come abbiamo detto - su Tabucchi, la penisola iberica e il portoghese, non mancano riflessioni che riguardano altri libri e altri temi, che tentano di chiarire altri lati della sfaccettata fisionomia di questo scrittore che deve alla sua complessità l'innegabile importanza che ha acquistato nel panorama letterario dell'ultimo cinquantennio. Se Isotta Piazza studia i personaggi lettori tra Piazza d'Italia e Requiem (personaggi lettori che permettono di sviluppare interessanti osservazioni sulla lettura e sulla letteratura viste dalla prospettiva tabucchiana e che fanno entrare nella raccolta anche alcune pagine del fondamentale Notturno indiano) e se il volume si 
chiude con un commosso ricordo di Giovanni Catelli (che è anche un omaggio allo scrittore di racconti e, in particolare, all'autore di Il gioco del rovescio e Piccoli equivoci senza importanza), William Spaggiari incentra il suo contributo sul Tabucchi che "ama giocare con le appropriazioni citazionistiche esplicite e dissimulate" e svolge un documentato percorso attraverso i riferimenti alla letteratura italiana contenuti nella narrativa tabucchiana, tra Leopardi (in La gastrite di Platone e Si sta facendo sempre più tardi, fino al "punto più alto del leopardismo di Tabucchi” rappresentato dal sogno del conte Giacomo in Sogni di sogni) e Montale: il Montale delle raccolte più canoniche, ma anche quello delle sillogi più tarde, che, da Satura, presta per esempio a Tabucchi l'immagine indimenticabile nel poeta ligure, ma anche nella rivisitazione tabucchiana - dell'angelo nero, inquietante presenza che affiora dal passato e che viene fissata in una delle pagine che - si presentino come narrazioni, saggi critici o interventi giornalistici - finiscono per andare a comporre il complessivo libro dell'inquietudine di un intellettuale e poeta della contemporaneità. GIOVANNI CAPECCHI
Parole per Antonio Tabucchi, a cura di Roberto Francavilla, Roma, Artemide, 2012, pp. 92.

Sembrerà forse bizzarro che la traduzione più plausibile della parola "tram" qui nel senese - dove sono nati $i$ contenuti che mi accingo a illustrare - sia "pollicino": espressione che indica, appunto, un piccolo bus di linea che percorre $\mathrm{i}$ vicoli del centro storico, arrancando per salite impervie, angusti vicoli di fattura medievale e scivolosi lastricati. Se vogliamo, si tratta di una variante toscana - e su gomma dell'omologo e sferragliante elétrico portoghese. L'occasione per questo futile paragone mi è offerta da uno scritto di Antonio Tabucchi dal titolo Il mio tram attraverso il Novecento, contenuto oggi nel prezioso volume Parole per Antonio Tabucchi che raccoglie, oltre a quattro inediti tabucchiani, l'omaggio di alcuni colleghi dell'Università di Siena all'amico e collega scomparso nel marzo del 2012. Dunque, d'ora in poi, appellandomi a modo mio al patto narrativo e con intento vagamente mimetico, vi chiedo di leggere "pollicino" laddove si scrive "tram", così per ricreare, almeno nel lessico, l'ambientazione geografica e culturale in cui queste 